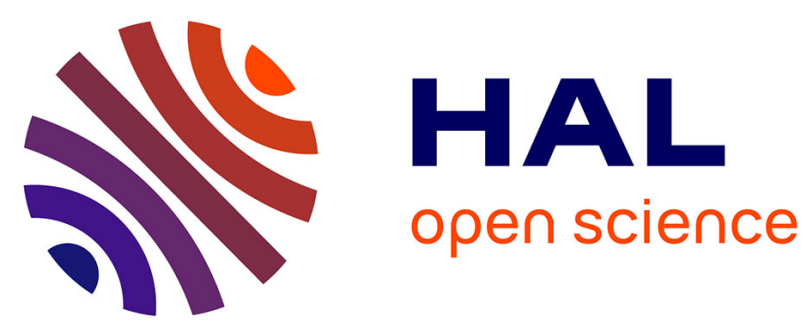

\title{
Méthodologie de conception de textures pour les interfaces tactiles à frottement programmable
} Ludovic Potier, Thomas Pietrzak, Géry Casiez, Nicolas Roussel

\section{To cite this version:}

Ludovic Potier, Thomas Pietrzak, Géry Casiez, Nicolas Roussel. Méthodologie de conception de textures pour les interfaces tactiles à frottement programmable. Ergo'IHM 2012 - Proceedings of the AFIHM Conférence Francophone sur l'interaction Homme-Machine, Oct 2012, Biarritz, France. pp.132-139, 10.1145/2652574.2653420 . hal-00719789

\section{HAL Id: hal-00719789 \\ https://hal.inria.fr/hal-00719789}

Submitted on 22 Jun 2018

HAL is a multi-disciplinary open access archive for the deposit and dissemination of scientific research documents, whether they are published or not. The documents may come from teaching and research institutions in France or abroad, or from public or private research centers.
L'archive ouverte pluridisciplinaire HAL, est destinée au dépôt et à la diffusion de documents scientifiques de niveau recherche, publiés ou non, émanant des établissements d'enseignement et de recherche français ou étrangers, des laboratoires publics ou privés. 


\section{Méthodologie de conception de textures pour les interfaces tactiles à frottement programmable}

\author{
Ludovic Potier \\ Inria Lille \\ ludovic.potier@inria.fr
}

\author{
Thomas Pietrzak \\ LIFL, Université de Lille \\ thomas.pietrzak@lifl.fr
}

\author{
Géry Casiez \\ LIFL, Université de Lille \\ gery.casiez@lifl.fr
}

\author{
Nicolas Roussel \\ Inria Lille \\ nicolas.roussel@inria.fr
}

\begin{abstract}
The design of textures for so-called variable friction technologies requires multiple perspectives, which this paper aims to outline and discuss. We first propose a definition of texture and describe the current state of knowledge on their perception. After presenting two technologies for variable friction and comparing them to other tactile interfaces, we describe several particular uses for these devices. We then discuss psychophysical methods for signal perception evaluation and finally discuss methodologies for creating multidimensional tactile content.
\end{abstract}

KEYWORDS: Tactile; variable roughness ; perception ; design methodology.

CATEGORIES AND SUBJECT DESCRIPTORS: H.5.2 [Information interfaces and presentation] : User interfaces : Haptic I/O, Evaluation/methodology

\section{INTRODUCTION}

Les technologies tactiles dites à frottement programmable sont de plus en plus étudiées en électronique et en interaction homme-machine. Elles offrent un grand potentiel en terme de flexibilité et de précision de la stimulation, donnant de nouvelles opportunités pour l'interaction tactile et gestuelle. Cependant, il n'existe pas aujourd'hui de méthode pour concevoir du contenu tactile adapté à ces dispositifs, où une approche multiple est nécessaire. La notion de texture semble être appropriée pour appréhender les différents niveaux de complexité des environnements tactiles. Son caractère multi-échelle peut être mis en parallèle des méthodologies permettant d'en évaluer la perception. De plus, ces textures sont spécifiques à la technologie tactile utilisée ; elles diffèrent à la fois au niveau des caractéristiques de la stimulation et au niveau de leur exploration.

Le but de ce travail est d'abord de proposer une taxonomie des technologies à frottement programmable, puis de faire une synthèse des méthodologies de conception des contenus tactiles. Dans une optique de conception, de dimensionnement et d'évaluation, nous détaillerons enfin la marche à suivre pour évaluer les différentes caractéristiques composant les textures ainsi que leur organisation en environnements tactiles cohérents.

\section{TEXTURES}

Nous allons d'abord nous intéresser au concept de texture, sachant qu'aucune définition générale n'existe aujourd'hui. Nous passerons ensuite en revue la littérature sur la perception des textures par le toucher. Bien que ces travaux s'intéressent essentiellement aux dimensions spatiales et vibratoires des textures [19], et que les dispositifs à frottement programmable permettent uniquement de faire varier le frottement, cela va être utile pour éclairer les mécanismes de la perception tactile des textures.

\section{Définitions}

Chaque discipline possède sa propre définition de la texture, qui est bien souvent spécifique au support ou au matériau étudié. En effet une texture en peinture est différente d'une texture en sciences des matériaux ou en imagerie numérique. Toutefois dans toutes ces définitions on retrouve un dénominateur commun : l'aspect multi-échelle de la texture (voir par exemple [42] pour l'alimentaire ou [16] pour le sonore).

Pour le toucher, nous appelons texture une séquence spatiale ou temporelle d'éléments singuliers (i.e. une forme distincte du fond), périodique ou non, formant un motif dont la complexité est variable (Figure 1). L'ordre de grandeur de ces éléments est inférieur à la taille d'un doigt pour qu'ils ne soient pas considérés comme des objets à part entière. Plusieurs motifs peuvent s'associer à des échelles différentes, procurant différents niveaux de détails à la texture, comme par exemple la rugosité due à la microstructure du matériau et l'organisation spatiale de picots en reliefs. La notion de 


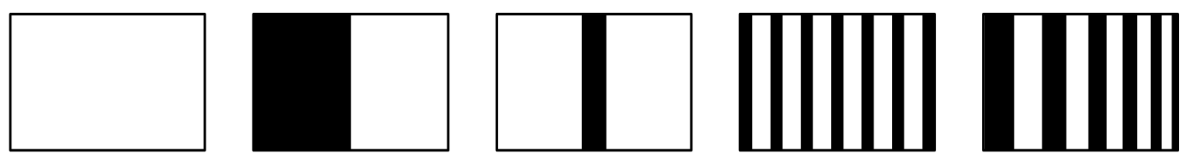

Figure 1 : Exemples de motifs de complexité croissante : signal, marche, forme, champ, gradient.

texture peut être envisagée dans un nombre quelconque de dimensions spatiales (typiquement 1,2 ou 3 ).

Le concept de textures amène donc à s'interroger sur les formes - spatiales ou temporelles - et plus généralement sur la perception de ces formes. Nous pourrions alors faire la distinction avec le concept d'icône, qui comme nous allons le voir par la suite, se base sur ces formes, mais dont l'étude pose cette fois des questions de sens, avec un objectif informatif. Ces deux dimensions cognitives de perception et de sens sont bien-sûr intimement liées, mais nécessitent des approches différentes pour leur étude.

\section{Perception des textures tactiles}

Le toucher permet une appréciation locale d'un objet, nécessairement située au point de rencontre entre le corps et la partie explorée. C'est la modalité perceptive du contact par excellence. Pour percevoir des caractéristiques globales d'un objet, comme sa forme ou sa texture, une exploration est donc nécessaire. Celle-ci peut prendre différentes formes, directement liées à la caractéristique explorée. On parle alors de procédures exploratoires [24, 15]. Par exemple, une pression tangentielle permet de percevoir la densité d'un matériau, ou encore un contact statique permet de percevoir sa température. C'est principalement le balayage latéral qui permet de percevoir la rugosité d'une surface.

Presque tous les travaux sur la perception des textures s'intéressent à la rugosité des matériaux [21]. Celle-ci correspond à l'échelle de la microstructure qui est aisément détectable par le toucher du fait de la résolution élevée de récepteurs de la peau, en particulier au bout des doigts. Deux types de stimulation contribuent à la perception de la rugosité : la variation spatiale de singularités et les vibrations [19]. Pour les textures très fines, ce sont surtout les vibrations qui permettent leur perception par le système haptique [18]. Une étude [29] a montré que la rugosité est invariante de la vitesse relative d'une surface mise en mouvement sur un doigt immobile, ce qui laisserait cette fois penser que la rugosité dépend des caractéristiques spatiales des reliefs (ici des picots). Dans ce sens, Cascio et Sathian [12] ont étudié l'influence de la taille des plateaux et des creux, ainsi que de leur fréquence de variation, dans la perception de la rugosité. Les auteurs établissent que c'est essentiellement la taille des creux qui influence la perception de la rugosité.
Nous savons déjà que l'utilisation d'un gradient de texture peut aider à reproduire une position ainsi qu'une distance [40]. D'autre part, dans une exploration libre, la perception de l'orientation d'une texture est possible en comparant celle-ci à une texture adjacente de référence [20]. Jin et Hughes [22] ont montré que même en compensant des gradients de texture à une dimension par des vitesses d'exploration relatives, afin de fournir une rugosité constante, les participants étaient toujours capables de détecter ces gradients. Il a aussi été montré qu'un gradient positif (organisé du moins dense au plus dense) permettait d'atteindre plus rapidement des cibles qu'avec un gradient négatif [37]. Ces textures étaient proposées dans un environnement numérique et leur exploration était réalisée à l'aide du dispositif de suppléance perceptive Tactos composé de cellules Braille. Enfin, nous savons qu'avec des interfaces à frottement programmable, les cibles possédant un coefficient de friction différent sont sélectionnées plus efficacement [13, 25].

Il pourrait être intéressant de reproduire ces expérimentations de psychologie expérimentale en utilisant les dispositifs à frottement programmable. Ceux-ci ne permettent pas d'afficher des textures en reliefs. Pourtant les travaux de Biet et al. [8] ont montré qu'une succession de zones rugueuses et lisses était perceptible comme une texture possédant une période spatiale.

Signalons aussi l'existence de recherches sur la génération automatique de textures et l'efficacité des algorithmes à produire des rugosités différentiables via une interface à retour de force [2]. Le but étant de distinguer au mieux des surfaces explorées par le toucher. Les textures produites ne possèdent aucune variation de structure dans l'espace ce qui limite leur caractère informatif. Le fait qu'elles n'aient pas de frontière nette entre les plateaux et les creux pourrait de plus les rendre difficiles à percevoir via une interface à frottement programmable.

\section{INTERFACES TACTILES}

Nous allons commencer par situer les interfaces à frottement programmable par rapport aux autres systèmes de retour tactile. Les trois principaux types d'interfaces tactiles diffèrent à la fois par leur technologie, mais aussi par leurs modes d'affichage (Table 1). 


\section{Vibreurs}

Les vibrations peuvent être obtenues principalement grâce à deux types de technologies : les moteurs à came décentrée et les actionneurs linéaires [31]. Quelle que soit la technologie, les vibrations générées sont tout de même audibles, surtout lorsque le dispositif est posé sur une surface résonnante comme le bois.

Les moteurs à came décentrée utilisent la force centrifuge pour produire une vibration lorsque le moteur tourne suffisamment rapidement. Ils se sont largement répandus avec l'essor des téléphones mobiles et des contrôleurs de jeux vibrants. Ils sont utilisés principalement pour avertir silencieusement l'utilisateur de l'arrivée d'un appel ou d'un message ou apporter des sensations tactiles dans un jeu. Leur intégration technologique est avancée du fait de leur industrialisation de masse, et leur consommation électrique est très faible. Cependant la qualité des effets produits est limitée à cause du délai d'activation et d'arrêt de l'ordre de plusieurs centaines de millisecondes.

Les actionneurs linéaires tels que le $\mathrm{C} 2$ de la société EAI, ou l'Haptuator [44] utilisent le même principe que les hautparleurs pour produire une vibration mécanique à l'aide d'un axe se déplaçant sous l'effet d'un champ magnétique créé par une bobine. Ils sont beaucoup plus réactifs que les moteurs à came décentrée, ce qui permet de contrôler finement l'effet rendu [11].

Les vibreurs sont des interfaces actives, c'est-à-dire que l'utilisateur perçoit les stimulations sans nécessité d'exploration; ils peuvent être pris en main ou placés sur le corps ou dans la poche.

\section{Matrices de picots}

Les matrices de picots permettent de commander de manière indépendante la montée ou la descente d'ergots motorisés, ce qui permet d'afficher des motifs tactiles évolués.

Leur usage est varié, depuis l'affichage de caractères Braille dans les claviers pour aveugles jusqu'à la simulation de textures [23] en passant par l'affichage d'icônes [35] ou d'images dans le cas de la substitution sensorielle [3]. La complexité mécanique de ces dispositifs génère des contraintes d'encombrement, particulièrement si l'on souhaite une résolution élevée. A titre d'exemple, le dispositif
Tactos [45] affichant 16 picots sur $1 \mathrm{~cm}^{2}$ tient dans $72 \mathrm{~cm}^{3}$ $\left(8 \times 3 \times 3 \mathrm{~cm}^{3}\right)$. Celui de Johnson et al. [23] affichant 400 picots sur $1 \mathrm{~cm}^{2}$ occupe lui un volume de $8 \mathrm{~m}^{3}(2 \times 2 \times 2 \mathrm{~m})$.

Ce type d'interface est soit perçu passivement, lorsque les matrices sont placées de manière immobile sur une partie du corps (i.e. on les porte), soit explorées activement, lorsqu'elles sont touchées par les utilisateurs [17].

\section{Frottement programmable}

Deux technologies existent aujourd'hui pour faire varier le frottement sur une surface : les électrovibrations et l'effet de film d'air comprimé. Bien que leur fonctionnement soit très différent, l'effet produit par ces deux technologies est très similaire; il consiste à faire varier le frottement sur un support et ainsi modifier les conditions de glissement des doigts.

Les plages de frottement disponibles sont relativement réduites, mais comme nous allons le voir plus loin, la frontière entre deux zones de frottements différents semble aisément perceptible. Ce sont des interfaces passives qui nécessitent l'exploration par le toucher pour percevoir l'information tactile. La modification du frottement agissant sur l'intégralité de la surface, on ne peut réellement parler de résolution de sortie mais si le contact avec la surface se limite à un point (e.g. un doigt), la résolution du retour produit devient de fait celle de la détection de ce point.

Si les principes de ces deux technologies sont bien connus et leur mise en œuvre maîtrisée, les recherches sur leurs usages pour l'interaction homme-machine en sont encore à leurs balbutiements. Avant de nous intéresser à ces recherches, nous allons décrire plus précisément le fonctionnement de ces dispositifs.

\section{DISPOSITIFS À FROTTEMENT PROGRAMMABLE}

La rugosité correspond à une propriété des matériaux relative à leur état de surface. Celle-ci influence le frottement, qui est une interaction physique entre deux surfaces en contact s'opposant à leur mouvement relatif [39]. Les dispositifs à frottement programmable permettent de moduler le frottement sur une même surface. Nous nous intéressons ici uniquement aux dispositifs intégrant la production d'un retour tactile au suivi de la position d'un ou plusieurs doigts,

\begin{tabular}{lcccccc}
\hline & Signal & Résol. $(\mathrm{mm})$ & Singularités & Intégration & Transparence & Réf. \\
\cline { 2 - 7 } Vibreurs & Actif & 0,5 & 1 & ++ & Oui & {$[11,44,31]$} \\
Matrice de picots & Actif/Passif & 1 & $\mathrm{n}$ & - & Non & {$[6,35,45]$} \\
Frott. prog. & Passif & Résol. input & 1 & + & Oui & {$[1,8]$} \\
\hline
\end{tabular}

Table 1 : Comparaison des trois principales technologies de retour tactile (ordres de grandeur). Résolution spatiale : taille du plus petit élément affichable. Singularités : nombre d'éléments différents affichables sous un doigt en même temps. Intégration technologique : miniaturisation du dispositif et facilité d'industrialisation. Transparence : possibilité de proposer le retour tactile sur un écran. 
condition nécessaire pour leur utilisation comme interface homme-machine.

\section{Électrovibration}

L'électrovibration permet d'augmenter la force normale entre un doigt et une surface, ce qui a pour effet d'augementer le frottement. Le principe consiste à créer une différence de potentiel électrique, le doigt et la surface pouvant être considérée comme les bornes d'un condensateur. Si un courant alternatif est appliqué à la surface conductrice, cela génère une force d'attraction intermittente entre le doigt et la surface. Cette force est trop faible pour être perçue de manière statique, mais elle permet de moduler le frottement entre la surface et la peau [41].

Utilisée par le dispositif TeslaTouch de Bau et al. [4], cette technologie est également commercialisée par la société Senseg. Elle a pour avantage de s'appliquer sur tout type de surface (plane ou non) avec un minimum de transformation (application d'un film conducteur transparent), et permet une sensation tactile uniforme sur toute la surface. Une limitation de ce système réside dans la nécessité de connecter le corps à la masse afin d'avoir un effet suffisant sans délivrer un voltage trop important. Bien que l'amplitude du courant soit faible, il est tout de même nécessaire d'appliquer une tension de $100 \mathrm{~V}$ au corps. Au-delà de l'impact possible sur la santé, cela rend l'intégration dans des appareils portatifs compliquée.

\section{Effet de film d'air comprimé}

Le principe de cette technologie consiste à générer un film d'air entre le doigt et la surface touchée afin de réduire le frottement sur celle-ci. Dans le cas du StimTac [1, 8], cet effet de film d'air comprimé (squeeze film effect) est produit par la vibration contrôlée de la surface à très haute fréquence par l'intermédiaire de céramiques piézoélectriques. Cette vibration dépassant les fréquences sensibles des méchanorécepteurs de la peau, ce n'est pas elle qui est ressentie, mais bien son effet sur la tribologie du contact. La surface concernée pouvant aussi bien être en métal qu'en verre, cette technologie peut être utilisée pour créer à la fois des pavés (touchpad) et des écrans tactiles.

Le LatPad [25] fonctionne de manière similaire au StimTac bien que son intégration soit moins avancée. Plus volumineux et plus bruyant, sa précision est également moindre en ce qui concerne la détection des contacts.

\section{Comparaison des deux approches}

La Table 2 résume les principales différences entre les deux technologies présentées (électrovibration et effet de film d'air comprimé). Comme nous l'avons vu, ces deux technologies produisent des effets contraires : l'une permet d'augmenter le frottement, l'autre de le réduire. Il pourrait ainsi être intéressant de coupler les deux technologies afin d'augmenter la plage de frottements possibles. Il est important de souligner que le frottement sur une surface est grandement influencée par les conditions atmosphériques (humidité, température) ainsi que par l'état de surface de la peau (humidité, propreté) [33] et ce de manière encore plus importante pour l'électrovibration où le contact entre la peau et la surface est aussi le lieu de passage du courant.

La commande nécessaire au contrôle des dispositifs basés sur ces deux technologies leur est spécifique. L'amplitude de vibration du StimTac est ainsi contrôlée par l'amplitude du courant d'alimentation des céramiques piézoélectriques. L'effet produit par TeslaTouch est lui piloté par la fréquence et l'amplitude du courant appliqué à la surface.Dans les deux cas, il est nécessaire de connaître la relation entre la commande et l'effet perçu par l'utilisateur. Pour ce faire et comme nous allons le voir, il est possible d'employer des méthodes de psychophysique.

Finalement, les dispositifs à frottement programmable fonctionnent comme des afficheurs de textures passifs : ils ne transfèrent pas d'énergie à l'utilisateur, mais modifient la manière dont l'énergie est dissipée au niveau de la surface de contact, lors d'un frottement provoqué par l'utilisateur. De tels dispositifs donnent la possibilité de générer des textures ou des reliefs statiques. Néanmoins, le fait de pouvoir piloter à volonté le signal ouvre de nouvelles voies pour produire du contenu tactile. En effet, les variations de frottement peuvent être spatialement fixes, tout comme elles peuvent être délivrées à intervalles de temps réguliers, ou encore relativement aux paramètres d'exploration. Ce type de dispositif semble être un outil puissant pour l'étude de la perception tactile, car il donne la possibilité de découpler actions et sensations, chose impossible dans une situation écologique (i.e. proche d'une situation d'usage).

\section{USAGES}

Les usages actuels des dispositifs à frottement programmable n'ont pas pour but de reproduire des textures réelles avec les caractéristiques de matériaux existants, mais plutôt

\begin{tabular}{lccccc}
\hline & Frottement & Transparence & Tension de fonctionnement (V) & Désagréments & Références \\
\cline { 2 - 6 } Électrovibration & Augmente & Oui & $80-120$ & Corps conducteur & {$[41,4]$} \\
Effet de film d'air & Réduit & Oui & 40 & Ultrasons & {$[8,1,25]$} \\
\hline
\end{tabular}

Table 2 : Comparaison des deux technologies de frottement programmable. 
de proposer aux utilisateurs un enrichissement de l'interaction ou de rendre accessibles certaines informations au travers du toucher. Ces dispositifs ont ainsi été principalement utilisés pour faciliter l'acquisition de cibles [13, 25].

D'autres pistes peuvent être envisagées pour tirer partie des possibilités des dispositifs à frottement programmable. Par exemple nous pouvons donner du relief à des icônes afin d'améliorer leur détection, ou bien proposer un motif tactile reflétant la fonction de chaque icône. L'utilisateur pourrait aussi consulter la présence de messages lorsque l'écran de l'appareil (téléphone ou ordinateur) est éteint, comme proposé par [26]. On pourrait envisager de permettre la distinction entre SMS et courriels, leur nombre, leur provenance ou leur niveau d'importance, par exemple. Par ailleurs, l'information est reçue à l'initiative de l'utilisateur, pas celle du système, ce qui évite d'être interrompu dans sa tâche. L'utilisateur pourrait aussi rendre accessible à ses interlocuteurs de messagerie instantanée son humeur du jour, par exemple lorsque ceux-ci touchent leur avatar, de manière similaire aux travaux utilisant les vibrations [38]. Nous pouvons aussi proposer des cartes géographiques augmentées de textures pour en faciliter la lecture ou l'accès par des personnes déficientes, de manière similaire à des systèmes à base de matrices de picots [34].

Afin de développer ces contenus tactiles, plusieurs étapes sont nécessaires. Il faut tout d'abord déterminer la relation entre le signal de commande et l'effet perçu. Une fois ces briques de bases connues, il sera possible de proposer des variations spatiales ou temporelles de frottement appropriées aux usages cités précédemment.

\section{MÉTHODES PSYCHOPHYSIQUES}

Les méthodes expérimentales pour évaluer la détection de signaux par un observateur constituent la psychophysique, inventée par Fechner au XIX ${ }^{\mathrm{e}}$ siècle pour relier le psychique interne au monde physique externe [43]. Le but peut être de déterminer des seuils de détection absolus, ou des seuils différentiels JND (just noticeable difference).

Ce type d'étude doit permettre de caractériser la relation entre le signal de l'interface à frottement programmable et l'effet ressenti par l'utilisateur. L'objectif étant d'obtenir des seuils nécessaires à la perception des éléments tactiles, à savoir le seuil de détection (seuil minimum à partir duquel une stimulation est perçue), le seuil de discrimination (valeur au delà de laquelle on ne perçoit plus de différence entre deux stimulations) et le seuil d'identification (valeur au delà de laquelle on n'est plus capable d'identifier une stimulation parmi plusieurs valeurs de références connues).

Biet et al. [8] ont ainsi réalisé une étude des seuils de discrimination de textures constituées de successions de zones à frottement élevé puis faible, et mises en œuvre à l'aide du StimTac. Celles-ci possédaient quatre périodes spatiales différentes. Une méthode à deux choix forcés (ou 2AFC, pour 2 alternative forced-choice) fut utilisée pour comparer les textures deux à deux. Les résultats montrent que le seuil de discrimination augmente avec la valeur de la période de référence et ce, de manière proportionnelle. Autrement dit, la fraction de Weber ${ }^{1}$ reste constante. L'autre résultat intéressant est que les participants arrivent à percevoir des textures dont la période spatiale est plus petite que la taille du doigt, ce qui est une illusion car les dispositifs à frottement programmable ne permettent de proposer qu'un signal unique sur toute leur surface à un instant donné.

Une étude psychophysique complète, incluant le seuil de détection ainsi que les seuils de discrimination, a aussi été menée sur le dispositif TeslaTouch [4]. Pour le seuil absolu, une méthode $2 \mathrm{AFC}$ a été utilisée. Une méthode 3AFC a été utilisée pour les seuils de discrimination. La connaissance des seuils de détection doit permettre d'optimiser la consommation du dispositif en ajustant l'intensité du signal au minimum perceptible. Les résultats sur les seuils différentiels ont permis de déterminer la granularité nécessaire pour concevoir deux sensations distinctes.

Ces deux études sont difficilement comparables, la première s'intéressant à des variations abruptes de frottement formant des textures, la seconde à des frottements absolus.

Nous voyons qu'une caractéristique intéressante de ces technologies, au-delà de la plage de frottement disponible qui est plus ou moins restreinte, réside dans la possibilité de faire varier ce frottement quasi-instantanément, du fait de leur haute fréquence de rafraîchissement. Cela permet ainsi de générer des marches qui semblent autrement plus saillantes que la variation progressive d'un frottement. Ce sont d'ailleurs ces marches qui pourront servir d'éléments de base pour la construction de textures étendues spatialement. Les méthodes psychophysiques sont finalement primordiales pour connaître le lien entre le signal de commande du dispositif et l'effet perçu par l'utilisateur, que l'on ne peut pas extrapoler du fait de la complexité de la chaîne entre la commande, les signaux électriques, les effets mécaniques et la perception par les différents utilisateurs. Les résultats obtenus par ces méthodes vont permettre de dimensionner les singularités formant les briques de bases des environnements tactiles.

\section{CONCEPTION MULTIDIMENSIONNELLE}

De nombreux travaux ont pour objectif de formaliser la conception d'icônes, en tant que vecteur de communication pour d'autres modalités que la vision. Bernsen [7] décrit l'icône comme constituée d'un média possédant quatre

\footnotetext{
1. Rapport de la plus petite différence perçue sur l'intensité du stimulus étalon
} 
types de propriétés : dynamique ou statique, correspondant à la dimension temporelle de la représentation ; linguistique ou non, correspondant aux éléments lexicaux (briques de base), syntaxiques (leur association) et sémantiques (signification) dans la représentation ; analogue ou non, correspondant à la ressemblance entre représentation et représenté ; arbitraire ou non, c'est-à-dire basée sur un système sémantique préexistant.

Ce sont principalement les aspects lexicaux et syntaxiques qui ont été étudiés dans les travaux mentionnés, afin de déterminer précisément la forme de ces icônes et en particulier la possibilité de distinguer les stimuli, la gestion de leur salience et de leur reconnaissabilité [27]. Il semble de plus important de créer un jeu d'icônes cohérent pour en faciliter l'apprentissage [36]. Les tactons sont des messages structurés et abstraits permettant de transmettre des informations de façon non visuelle [9]. Leur structure est inspirée d'icônes sonores [10], qui permet de représenter des informations de manière hiérarchique à l'aide de paramètres tels que : le rythme, la durée ainsi que le tempo, qui doivent être différenciés au maximum; le frottement, créé à partir de divers paramètres tels que la modulation de fréquence ou d'amplitude; la forme du signal : sinusoïdal, carré, triangulaire, etc. ; la localisation spatiale, créée en utilisant plusieurs vibreurs. Ce concept a été utilisé par d'autres types de retours haptiques. Par exemple les icônes sur matrices de picots $[35,36]$, les paramètres utilisés sont : le type de motif, sa taille et la vitesse d'affichage.

La difficulté qui se pose avec l'utilisation d'un grand nombre de paramètres est de savoir comment les faire varier pour proposer un jeu où chaque icône est saillante et différentiable des autres. Plusieurs méthodes permettent d'évaluer les icônes multi-dimensionnelles, dont la méthode de multidimensional scaling (MDS) et la réduction d'incertitude.

Le MDS permet d'extraire les axes perceptifs d'icônes possédant de nombreux paramètres, bien que son interprétation soit délicate en l'absence de statistiques [28]. MDS fonctionne comme un outil de visualisation permettant de révéler des structures à partir d'une matrice de dissimilarité entre les stimuli. Ces dissimilarités peuvent être évaluées soit une par une, ce qui est très long, soit en utilisant une méthode de classification (cluster sorting method) où les participants ont pour tâche de ranger les stimuli dans des classes et ce, à plusieurs reprises et avec un nombre de classes différent. On compte ensuite le nombre de fois où une paire a été rassemblée, ce qui permet de créer la matrice de dissimilarité. Deux types d'algorithmes existent pour la visualisation des résultats de MDS : les métriques (qui conservent les distances entre les paires) et les non-métriques (qui conservent le rang entre les paires). Suivant le nombre de paramètres tracés, de nouveaux éléments peuvent être mis en évidence [32].
D'autres types de visualisations peuvent être associés en complément, comme la technique du pathfinder ou la classification ascendante hiérarchique [27].

La méthode de réduction d'incertitude (MRI) a été utilisée dans plusieurs études sur les icônes tactiles [11,35], et se base sur la théorie de l'information. Le principe consiste à mesurer la différence entre la quantité d'information du stimulus et la quantité d'information reçue par l'utilisateur [30] : la quantité d'information du stimulus se mesure au nombre de comparaisons nécessaires pour identifier la bonne icône, en supposant qu'on sache toutes les différencier deux à deux et ce, grâce à l'analyse des confusions. Ainsi, si pour un même stimulus la variété des réponses données est grande, il y a peu d'information transmise. Si au contraire pour un stimulus donné la réponse est toujours la même, toute l'information est transmise. Bien qu'étant généralement considérées comme des erreurs, si d'autres réponses sont fréquentes, elles contiennent de l'information, qui correspond à la similarité entre les icônes en question.

Une fois le jeu d'icônes réalisé, il est possible d'évaluer sa capacité a être appris et mémorisé dans des conditions plus ou moins écologiques [14]. Il peut aussi être intéressant de vérifier l'efficacité de l'association entre une icône signifiante et l'objet signifié $[11,36]$.

Dans la plupart ces travaux, les utilisateurs sont donc passifs lors de la perception des stimuli. Le terme passif est à entendre ici dans le sens où aucun mouvement exploratoire n'est réalisé, il ne prend pas en compte le caractère actif de l'implication du sujet et de la focalisation de son attention. Comme nous l'avons vu, les dispositifs à frottement programmable exigent une exploration active. L'avantage de pouvoir délivrer un signal spatial ou temporel, et de pouvoir mesurer l'exploration des utilisateurs est de disposer d'un grand potentiel de paramètres pour la création d'icônes. Le désavantage est que la conception de contenu tactile est plus difficile.

Finalement, toutes ces méthodes semblent pouvoir être utilisées pour la conception d'environnements tactiles destinés aux dispositifs à frottement programmable. Il semble nécessaire de respecter un certain ordre, afin de travailler sur des éléments tactiles de plus en plus complexes (Table 3).

\section{CONCLUSION}

Les dispositifs à frottement programmable offrent une grande flexibilité pour la conception d'environnements tactiles complexes. Ils permettent de générer des textures à dimensions multiples, pouvant s'organiser en sets d'icônes cohérents, afin de donner accès aux environnements numériques par le biais du toucher.

Nous avons passé en revue les moyens techniques existants pour moduler le frottement d'une interface, leurs contraintes 


\begin{tabular}{lccc}
\hline & 1. Signal & 2. Texture & 3. Icône \\
\cline { 2 - 4 } Test & Détect., Discrim., Ident. & Qualités, Orientation, Pointage & Signification, Cohérence \\
Méthode & Psychophysique & Psychologie expérimentale & MDS, MRI \\
\hline
\end{tabular}

Table 3 : Synthèse de la méthodologie à mettre en œuvre pour évaluer les différentes caractéristiques des éléments tactiles.

et leurs opportunités pour la conception et le dimensionnement d'environnements tactiles numériques, ainsi que leurs différences fondamentales avec les autres systèmes à retour tactile. Les méthodes psychophysiques permettent d'évaluer précisément la relation entre la valeur du signal et sa détection ou sa discrimination avec un autre signal. À partir de ces règles de conception de base, il est possible de construire une grande variété d'éléments, composés de variations de frottement dans l'espace et dans le temps. Ces textures organisées dans l'espace peuvent alors avoir de nouvelles caractéristiques de frottement, mais aussi des propriétés d'orientation ou de variation de densité. Elles forment des icônes tactiles pouvant s'organiser en un ensemble cohérent pour une situation d'usage donnée. Ce travail de conception doit être mené de manière spécifique pour s'adapter aux spécificités des dispositifs à frottement programmable, et prendre ainsi en compte les paramètres d'action de l'utilisateur. Comme proposé par Beaudouin-Lafon [5], cette tâche consiste en la conception d'interactions, en tant que phénomènes sensorimoteurs relatifs à une activité avec un produit, et non uniquement du design d'interface.

Pour finir, la question de la relation entre le mouvement d'exploration et la perception des formes tactiles mène à un vaste champ de recherche en sciences cognitives. La flexibilité et la précision des dispositifs à frottement programmable pourrait être très utile à l'étude du couplage sensorimoteur, intrinsèque à la perception, avec la possibilité nouvelle de pouvoir dé-corréler les sensations tactiles des actions réalisées durant l'exploration.

\section{BIBLIOGRAPHIE}

1. Amberg, M., Giraud, F., Lemaire-Semail, B., Olivo, P., Casiez, G., and Roussel, N. Stimtac, a tactile input device with programmable friction. In Proc. of UIST '11, 7-8. ACM, 2011.

2. Aysal, T. C., and Barner, K. E. Stochastic and deterministic models for haptic pseudo-textures. In Proc. of Haptics '06, 71-79. IEEE, 2006.

3. Bach-Y-Rita, P. Brain Mechanisms in Sensory Substitution. New York : Academic Press, 1972.

4. Bau, O., Poupyrev, I., Israr, A., and Harrison, C. Teslatouch : electrovibration for touch surfaces. In Proc. of UIST '10, 283-292. ACM, 2010.

5. Beaudouin-Lafon, M. Designing interaction, not interfaces. In Proc. of AVI '04, 15-22, 2004.
6. Benali Khoudja, M., Sautour, A., and Hafez, M. Tactile feedback : Towards a new tactile language to communicate emotions. In Proc. of Virtual Concept '05, 2005.

7. Bernsen, N. O. Modality theory in support of multimodal interface design. In ERCIM Workshop on Multimodal HMI, 37-44, 1993.

8. Biet, M., Casiez, G., Giraud, F., and Lemaire-Semail, B. Discrimination of virtual square gratings by dynamic touch on friction based tactile displays. In Proc. of Haptics '08, 41-48. IEEE, 2008.

9. Brewster, S., and Brown, L. M. Tactons : structured tactile messages for non-visual information display. In Proc. of AUIC '04, 15-23, 2004.

10. Brewster, S. A., Wright, P. C., and Edwards, A. D. N. Experimentally derived guidelines for the creation of earcons. In Proc. of BCS-HCI '95, 1995.

11. Brown, L. M., Brewster, S. A., and Purchase, H. C. A First Investigation into the Effectiveness of Tactons. In Proc. of WHC '05, 167-176. IEEE, 2005.

12. Cascio, C. J., and Sathian, K. Temporal cues contribute to tactile perception of roughness. The Journal of Neurosciences, 21 :5289 - 5296, 2001.

13. Casiez, G., Roussel, N., Vanbelleghem, R., and Giraud, F. Efficacité et robustesse aux distracteurs d'un retour tactile pour faciliter le pointage. In Proc. of IHM '10, 25-32. ACM, 2010.

14. Chan, A., Maclean, K., and Mcgrenere, J. Learning and identifying haptic icons under workload. In Proc. of Eurohaptics '05, 432-439, 2005.

15. Cooke, T., Wallraven, C., and Bülthoff, H. H. Multidimensional scaling analysis of haptic exploratory procedures. volume $7,7: 1-7: 17$. ACM, 2010.

16. Dubnov, S., Bar-Joseph, Z., El-Yaniv, R., Lischinski, D., and Werman, M. Synthesizing sound textures through wavelet tree learning. Computer Graphics and Applications, 38-48, 2002.

17. Hayward, V., Astley, O. R., Cruz-Hernandez, M., Grant, D., and Robles-de-la Torre, G. Haptic interfaces and devices. Sensor Review, 24 :16-29, 2004. 
18. Hollins, M., Bensmaia, S. J., and Roy, E. A. Vibrotaction and texture perception. Behavioural Brain Research, 135 :51 - 56, 2002.

19. Hollins, M., and Risner, S. R. Evidence for the duplex theory of tactile texture perception. Perception and Psychophysics, 62 :695 - 705, 2000.

20. Hughes, B. Haptic exploration and the perception of texture orientations. Haptics-e, 4, 2006.

21. Hughes, B., Wang, J., Rosic, D., and Palmer, K. Texture gradients and perceptual constancy under haptic exploration. In Proc. of WHC '07, 66-71. IEEE, 2007.

22. Jin, W., and Hughes, B. Effect of density gradients on haptic discrimination. Acta Psychologica Sinica, $37: 739$ - 747, 2005.

23. Killebrew, J. H., Bensmaia, S. J., Dammann, J. F., Peter Denchev, P., Hsiao, S. S., Craig, J. C., and Johnson, K. O. A dense array stimulator to generate arbitrary spatio-temporal tactile stimuli. Journal of Neurosciences Methods, 161 :62-74, 2007.

24. Lederman, S. J., and Klatzky, R. L. Hand movements : A window into haptic object recognition. Cognitive Psychology, 19 :342-368, 1987.

25. Levesque, V., Oram, L., MacLean, K., Cockburn, A., Marchuk, N. D., Johnson, D., Colgate, J. E., and Peshkin, M. A. Enhancing physicality in touch interaction with programmable friction. In Proc. of CHI' 11, 2481-2490. ACM, 2011.

26. Luk, J., Pasquero, J., Little, S., and Maclean, K. A role for haptics in mobile interaction : initial design using a handheld tactile display prototype. In Proc. of CHI '06, 171-180. ACM, 2006.

27. MacLean, K. Foundations of transparency in tactile information design. IEEE Trans. on Haptics, 1(2) :84 $-95,2008$.

28. Maclean, K., and Enriquez, M. Perceptual design of haptic icons. In Proc. of Eurohaptics '03, 351-363, 2003.

29. Merftah, E. M., Belingard, L., and Chapman, C. E. Relative effect of the spatial and temporal characteristics of scanned surfaces on human perception of tactile roughness using passive touch. Experimental Brain Research, 132 :351 - 361, 2000.

30. Miller, G. A. The magical number seven, plus or minus two : Some limits on our capacity for processing information. Psychological Review, 63 :81-97, 1956.

31. Mortimer, B. J. P., Zets, G. A., and Cholewiak, R. W. Vibrotactile transduction and transducers. Journal of the Acoustical Society, 121 :2970-2977, 2007.
32. Pasquero, J., Luk, J., Little, S., and Maclean, K. Perceptual analysis of haptic icons : an investigation into the validity of cluster sorted mds. In Proc. of Haptics '06. IEEE, 2006.

33. Pasumarty, S., Johnson, S., Watson, S., and Adams, M. Friction of the human finger pad : Influence of moisture, occlusion and velocity. Tribology Letters, 44 :117-137, 2011.

34. Petit, G., Dufresne, A., Levesque, V., Hayward, V., and Trudeau, N. Graphisme tactile appliqué aux illustrations de manuels scolaires à l'usage d'enfants ayant une déficience visuelle. In Proc. IHM 2008, 73-80, New York, NY, USA, 2008. ACM.

35. Pietrzak, T., Crossan, A., Brewster, S. A., Martin, B., and Pecci, I. Creating usable pin array tactons for non-visual information. IEEE Trans. on Haptics, 2(2) :61-72, 2009.

36. Pietrzak, T., Pecci, I., and Martin, B. Static and dynamic tactile directional cues experiments with vtplayer mouse. In Proc. of Eurohaptics '06, 63-68, 2006.

37. Potier, L., Gapenne, O., Liu, X., and Aubert, D. The effect of the direction of a gradient on haptic pointing in a $2 \mathrm{~d}$ virtual environment. In Proc. of IADIS Interfaces and HCI'11, 2011.

38. Rovers, A., and van Essen, H. Him : a framework for haptic instant messaging. In Proc. of CHI 'O4, 1313-1316. ACM, 2004.

39. Sahin, M., Çetinarslan, C. S., and Erol Akata, H. Effect of surface roughness on friction coefficients during upsetting processes for different materials. Materials and Design, 28 :633-640, 2005.

40. Schellingerhout, R., Smitsman, A. W., and Van Galen, G. P. Temporal cues contribute to tactile perception of roughness. Acta Psychologica, 99 :93 - 114, 1998.

41. Strong, R. M., and Troxel, D. E. An electrotactile display. In Trans. on Man-Machine Systems, IEEE, 72-79, 1970.

42. Szczesniak, A. S. Texture is a sensory property. Food Quality and Preference, 215-225, 2002.

43. Treutwein, B. Adaptative psychophysical procedures. Vision Research, 35(17) :2503-2522, 1995.

44. Yao, H.-Y., and Hayward, V. Design and analysis of a recoil-type vibrotactile transducer. Journal of the Acoustical Society, 128 :619-627, 2010.

45. Ziat, M., Gapenne, O., Stewart, J., and Lenay, C. Haptic recognition of shapes at different scales : A comparison of two methods of interaction. Interacting with Computers, 19 :121-132, 2007. 